\title{
Explicit solution for the natural frequency of structures with partial viscoelastic treatment
}

\section{Høgsberg, Jan Becker}

\section{Published in:}

Mechanics of Advanced Materials and Structures

Link to article, DOI:

10.1080/15376494.2015.1029166

Publication date:

2016

Document Version

Peer reviewed version

Link back to DTU Orbit

Citation (APA):

Høgsberg, J. B. (2016). Explicit solution for the natural frequency of structures with partial viscoelastic treatment. Mechanics of Advanced Materials and Structures, 23(7), 784-790.

https://doi.org/10.1080/15376494.2015.1029166

\section{General rights}

Copyright and moral rights for the publications made accessible in the public portal are retained by the authors and/or other copyright owners and it is a condition of accessing publications that users recognise and abide by the legal requirements associated with these rights.

- Users may download and print one copy of any publication from the public portal for the purpose of private study or research.

- You may not further distribute the material or use it for any profit-making activity or commercial gain

- You may freely distribute the URL identifying the publication in the public portal

If you believe that this document breaches copyright please contact us providing details, and we will remove access to the work immediately and investigate your claim. 


\title{
EXPLICIT SOLUTION FOR THE NATURAL FREQUENCY OF STRUCTURES WITH PARTIAL VISCOELASTIC TREATMENT
}

\author{
JAN HøGSBERG \\ jhg@mek.dtu.dk \\ Department of Mechanical Engineering, \\ Technical University of Denmark, 2800 Kongens Lyngby, Denmark
}

\begin{abstract}
The free vibration characteristics of structures with viscoelastic treatment are represented by the complex-valued natural frequencies. The assumed single mode representation associated with the low-frequency stiffness of the viscoelastic treatment is modified by a correction term representing the influence from residual vibration modes. The correction term is eliminated in terms of the corresponding natural frequency associated with the high-frequency stiffness of the viscoelastic treatment, whereby an expression is obtained for the complex-valued natural frequency, which only requires the solution of two real-valued eigenvalue problems.
\end{abstract}

\section{INTRODUCTION}

While the introduction of supplemental viscoelastic material in many engineering problems is a robust approach to vibration mitigation of flexible structures, the desire to add substantial attainable damping relies on the inherent compromise between the magnitude of the stiffness and the energy dissipation properties of the viscoelastic material. Thus, the design of viscoelastic treatment for damping of flexible structures requires an accurate estimation of the damping level, which again requires the solution of the full eigenvalue problem in state-space with relaxation properties governed by additional state variables or by an iterative procedure with a frequency dependent dynamic stiffness matrix $[1,2]$.

Viscoelastic treatment is in many cases introduced as dissipative elements in passive or passive-active sandwich beams [3,4] or in terms of constrained layer damping systems [5-7] where energy dissipation is obtained by shear deformation of the viscoelastic material. Full coverage of the structure by viscoelastic treatment may be unfeasible and as demonstrated in [8] partial coverage may be nearly as effective, as long as the treatment is placed where 
the deformation of the targeted vibration form of the structure is substantial [7]. The concepts for numerical modeling of a flexible structure with viscoelastic treatment are numerous, and the inherent compromise between accuracy and computational effort has been a main aspect in the development of the various proposed solution strategies. The most common modeling approaches are assumed modal expansions [7-11] or discretization by finite elements $[4,12,13]$. Both modeling approaches lead to discretized equations of motion, where the complex-valued natural frequencies are determined by solving the full dimensional eigenvalue problem. Concepts have recently been proposed to determine the complex-valued natural frequencies of dynamic systems with supplemental viscoelastic attachments [14] and structures with integrated viscoelastic parts [11,15]. A review of numerical solution strategies for solving the damped eigenvalue problem for viscoelastic plate structures is given in [16].

The aim of the present paper is to establish a simple, explicit, accurate and cost-effective solution format for determining the complex-valued natural frequencies of flexible structures with partially distributed linear-viscoelastic treatment. The viscoelastic material is assumed to be of relaxation form, with real-valued elastic properties in the low- and high-frequency limits and with frequency dependent properties representing the time relaxation. In Section 2 the relaxation format is initially introduced and the discretized equations of motion of the structure with partial viscoelastic treatment are presented. The modal properties of the flexible structure are then defined with respect to both the lowand the high-frequency stiffness contributions from the viscoelastic treatment. In Setion 3 the modal scalar equations for the resonant vibration form of the structure are now determined, where an essential part of the present solution strategy is the introduction of an additional term, representing the modal flexibility from non-resonant residual vibration modes. This type of correction term is commonly applied in numerical analysis for effective truncation of series expansions [17-19]. But recently this approach has also been used in $[20,21]$ to derive accurate calibration formulae for resonant vibration damping of flexible structures. A compact solution format for the complex-valued natural frequency of the resonant vibration mode is derived from the scalar equations of motion, and an optimality condition is finally formulated for maximum damping of the flexible structure. 
The accuracy of the frequency solution format is then investigated by a simple numerical example in Section 4.

\section{Structure With Viscoelastic treatment}

2.1. Relaxation material model. Constitutive relations for viscoelastic materials are conveniently described by linear models, where the stress components are governed by a convolution integral with a decaying exponential kernel representing strain relaxation $[22,23]$. In the frequency domain, with angular frequency $\omega$, these linear relaxation characteristics imply real-valued material stiffness in both the low- and high-frequency limits. In a one dimensional relaxation model the constitutive relation can therefore be written as

$$
\sigma(\omega)=E(\omega) \varepsilon=\left(E_{0}+\Delta E G(\omega)\right) \varepsilon
$$

where $\sigma$ and $\varepsilon$ are the energy conjugated stress and strain components, respectively. The frequency dependent properties of the viscoelastic modulus $E(\omega)$ is represented by the transfer function $G(\omega)$, with defined low- and high-frequency limits $G(0)=0$ and $G(\infty)=$ 1 , respectively. Thus, the viscoelastic modulus recovers $E(0)=E_{0}$ in the low-frequency limit and $E(\infty)=E_{0}+\Delta E$ in the high-frequency regime, whereby $\Delta E$ represents the change in stiffness between the two frequency limits. As demonstrated in [24,25] causality implies $\Delta E \geq 0$. Figure 1 shows the real and imaginary part of $G(\omega)=i \omega \tau /(1+i \omega \tau)$ for the standard three-parameter relaxation model [26], in which the relaxation time scale $\tau$ determines the transition frequency between the low- and high-frequency domain.

2.2. Discrete equations of motion. Flexible structures with partial viscoelastic treatment are commonly discretized by finite elements or by a truncated series of orthogonal functions. In a frequency domain representation of the system of governing equilibrium equations the discretized numerical model can therefore be written as

$$
\left(-\omega^{2} \mathbf{M}+\mathbf{K}\right) \mathbf{u}=-\mathbf{H} \Delta \mathbf{f}
$$

where the resulting stiffness matrix $\mathbf{K}=\mathbf{K}_{s}+\mathbf{H k}_{0} \mathbf{H}^{T}$ in this case comprises the stiffness matrix $\mathbf{K}_{s}$ from the structure without the viscoelastic part and the stiffness matrix $\mathbf{H k}_{0} \mathbf{H}^{T}$ associated with the low-frequency viscoelastic stiffness $E_{0}$. The participation array $\mathbf{H}$ defines the reduced number of degrees of freedom that describe the motion of the viscoelastic 

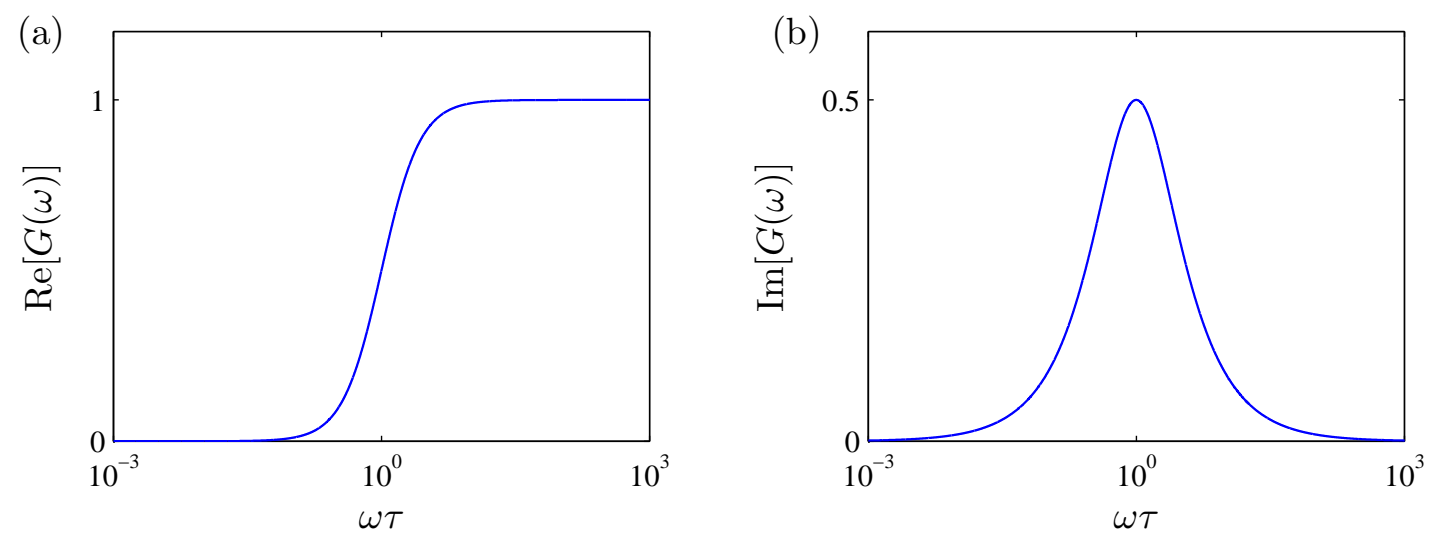

FiguRE 1. Real (a) and imaginary part (b) of frequency dependent function $G(\omega)$ for three-parameter relaxation model.

treatment by the relation

$$
\mathbf{v}=\mathbf{H u}
$$

Thus, the matrix $\mathbf{k}_{0}$ represents the stiffness associated with the viscoelastic degrees of freedom in $\mathbf{v}$, while $\mathbf{H k}_{0} \mathbf{H}^{T}$ is the corresponding stiffness matrix expanded to full model dimensions by the participation array $\mathbf{H}$. In Eq. (2) the mass matrix $\mathbf{M}$ represents the combined inertia from the structure and the viscoelastic treatment.

On the right hand side of Eq. (2) the vector $\Delta \mathbf{f}$ represents the additional contribution from the viscoelastic treatment relative to the low-frequency stiffness. The force increment $\Delta \mathbf{f}$ is energetically conjugate to the reduced displacement vector $\mathbf{v}$ and is therefore expanded to full model dimensions in Eq. (2) by the pre-multiplication with $\mathbf{H}$. To simplify the outline of the paper it is assumed that the frequency dependent properties of the viscoelastic material are constant with respect to the spatial distribution of the treatment, whereby the frequency dependent function $G(\omega)$ defined in Eq. (1) remains a scalar function in the discretized numerical model. Thus, the incremental force vector on the right hand side of Eq. (2) can be written as

$$
\Delta \mathbf{f}=G(\omega) \Delta \mathbf{k v}
$$


where the stiffness matrix $\Delta \mathrm{k}$ is derived from the elastic increment $\Delta E$ between the lowand high-frequency limits, whereby the force increment $\Delta \mathbf{f}$ represents the contribution from the stress increment $\Delta E G(\omega) \varepsilon$.

2.3. Modal properties. The complex frequency analysis in Section 3 is based on a modal representation of the structural response. In the governing equation of motion (2) the stiffness matrix contains the low-frequency stiffness contribution from the viscoelastic treatment. In the low-frequency domain $G(0)=0$, whereby Eq. (4) gives $\Delta \mathbf{f}=\mathbf{0}$. Thus, in the low-frequency limit Eq. (2) corresponds to the real-valued eigenvalue problem

$$
\left(\mathbf{K}-\omega_{j}^{2} \mathbf{M}\right) \mathbf{u}_{j}=\mathbf{0}
$$

with the square of the natural frequency $\omega_{j}^{2}$ as the eigenvalue and the mode shape $\mathbf{u}_{j}$ as the eigenvector. The modal mass and stiffness are in the following defined as $m_{j}=\mathbf{u}_{j}^{T} \mathbf{M} \mathbf{u}_{j}$ and $k_{j}=\mathbf{u}_{j}^{T} \mathbf{k} \mathbf{u}_{j}$, respectively, and the natural frequency in Eq. (5) is therefore defined by the ratio $\omega_{j}^{2}=k_{j} / m_{j}$.

In the high-frequency limit the frequency dependent function $G(\infty)=1$, whereby Eq. (4) gives $\Delta \mathbf{f}=\Delta \mathbf{k v}$. Substitution of this stiffness relation into Eq. (2) gives the alternative real-valued eigenvalue problem

$$
\left(\mathbf{K}+\mathbf{H} \Delta \mathbf{k} \mathbf{H}^{T}-\left(\omega_{j}^{2}+\Delta \omega_{j}^{2}\right) \mathbf{M}\right)(\mathbf{u}+\Delta \mathbf{u})_{j}=\mathbf{0}
$$

corresponding to the high-frequency limit of the viscoelastic material stiffness. The eigensolution is expressed in terms of the change in the square of the natural frequency $\Delta \omega_{j}^{2}$ and the corresponding change in the mode shape vector $\Delta \mathbf{u}_{j}$, relative to the eigensolution $\left(\omega_{j}^{2}, \mathbf{u}_{j}\right)$ obtained in Eq. (5). Both Eqs. (5) and (6) are real-valued eigenvalue problems that are effectively solved to determine the corresponding natural frequencies and mode shape vectors.

\section{NATURAL FREQUENCY SOLUTiON}

3.1. Modal equations. The reduced displacement vector $\mathbf{v}$ in Eq. (4) receives contributions from all vibration modes of the structure with viscoelastic treatment. In the present case the displacement vector is approximated by the mode shape vector of the resonant 
vibration form $(j=r)$ and an additional flexibility contribution from the residual nonresonant vibration modes $(j \neq r)$. Following the approach in $[20,21]$ the deformation of the viscoelastic part in Eq. (4) can therefore be represented as

$$
\mathbf{v}=\mathbf{v}_{r} q_{r}-\frac{1}{k_{0}}\left(\mathbf{v}_{r} \mathbf{v}_{r}^{T}\right) \Delta \mathbf{f}
$$

where $q_{r}$ is the modal coordinate associated with the resonant mode shape $\mathbf{u}_{r}$, while the vector $\mathbf{v}_{r}=\mathbf{H}^{T} \mathbf{u}_{r}$ represents the corresponding modal displacement of the viscoelastic part of the structure. In Eq. (7) the parameter $1 / k_{0}$ represents the additional flexibility from the non-resonant vibration modes of the structure. The assumed single mode representation is therefore retained by $1 / k_{0}=0$. In the force relation (4) the viscoelastic displacement $\mathbf{v}$ is now eliminated by Eq. (7). This gives

$$
\frac{1}{k_{r}} \mathbf{v}_{r}^{T} \Delta \mathbf{f}=\frac{G(\omega) \Delta k_{r} / k_{r}}{1+G(\omega) \Delta k_{r} / k_{0}} q_{r}
$$

where the modal stiffness increment is defined as

$$
\Delta k_{r}=\mathbf{v}_{r}^{T} \Delta \mathbf{k} \mathbf{v}_{r}
$$

The modal representation of the structural equation of motion (2) is

$$
\left(-\omega^{2}+\omega_{r}^{2}\right) q_{r}=-\frac{\omega_{r}^{2}}{k_{r}} \mathbf{v}_{r}^{T} \Delta \mathbf{f}
$$

and Eqs. (10) and (8) constitute the two coupled scalar equations for the resonant vibration mode $j=r$, which together determine the complex-valued natural frequency $\omega$ of the structure with partial viscoelastic treatment.

3.2. General solution format. Elimination of $\mathbf{v}_{r}^{T} \Delta \mathbf{f} / k_{r}$ between Eqs. (10) and (8) gives the frequency transfer relation

$$
\left(-\omega^{2}+\omega_{r}^{2}\left[1+\frac{\frac{\Delta k_{r}}{k_{r}} G(\omega)}{1+\frac{\Delta k_{r}}{k_{0}} G(\omega)}\right]\right) u_{r}=0
$$

where the free vibration properties are governed by the characteristic equation associated with the parenthesis on the left hand side. A solution for the complex natural frequency $\omega$ is conveniently expressed in terms of the increment $\Delta \omega^{2}=\omega^{2}-\omega_{r}^{2}$, relative to the natural 
frequency $\omega_{r}$ governed by the eigenvalue problem (5). This solution can be written as

$$
\frac{\Delta \omega^{2}}{\omega_{r}^{2}}=\frac{\frac{\Delta k_{r}}{k_{r}} G(\omega)}{1+\frac{\Delta k_{r}}{k_{0}} G(\omega)}
$$

It is seen that this expression recovers the solution $\omega=\omega_{r}$ in the low-frequency limit with $G(0)=0$. In the corresponding high-frequency limit with $G(\infty)=1$ the solution in Eq. (12) gives

$$
\frac{\Delta \omega_{r}^{2}}{\omega_{r}^{2}}=\frac{\frac{\Delta k_{r}}{k_{r}}}{1+\frac{\Delta k_{r}}{k_{0}}}
$$

where the increment $\Delta \omega_{r}^{2}$ is determined by the associated eigenvalue problem in Eq. (6). Thus, the non-dimensional flexibility parameter $\Delta k_{r} / k_{0}$ can now be obtained from Eq. (13) and substituted into the frequency solution in Eq. (12). This gives

$$
\frac{\Delta \omega^{2}}{\omega_{r}^{2}}=\frac{\frac{\Delta k_{r}}{k_{r}} G(\omega)}{1+G(\omega)\left(\frac{\omega_{r}^{2}}{\Delta \omega_{r}^{2}} \frac{\Delta k_{r}}{k_{r}}-1\right)}
$$

where $\omega_{r}^{2}$ and $\Delta \omega_{r}^{2}$ are determined by the real-valued eigenvalue problems (5) and (6), respectively. The solution in Eq. (14) can be written in compact form as

$$
\frac{\Delta \omega^{2}}{\Delta \omega_{r}^{2}}=\frac{\eta_{r}(\omega)}{1+\eta_{r}(\omega)}
$$

introducing the generalized frequency dependent parameter

$$
\eta_{r}(\omega)=\frac{\Delta k_{r}}{k_{r}} \frac{\omega_{r}^{2}}{\Delta \omega_{r}^{2}} \frac{G(\omega)}{1-G(\omega)}
$$

The solution in Eq. (15) becomes explicit when assuming that the frequency dependency is evaluated at the resonance frequency, whereby $\eta_{r}(\omega) \simeq \eta_{r}\left(\omega_{r}\right)$. As demonstrated in the following numerical example this explicit form of the solution in Eq. (15) gives good agreement with the exact numerical solution. Furthermore, it is enables the determination of criteria for optimal damping of the flexible structure by the viscoelastic treatment. An expression similar to Eq. (15) has been derived for structures with viscous dampers in [27] or with linear active control in [28], based on a two-component representation of the 
damped vibration mode. In the present case this extended modal representation is directly contained in the correction term with $1 / k_{0}$ in Eq. (7).

3.3. Optimality condition. Maximum attainable damping is obtained at the corresponding maximum of the imaginary part of the complex-valued natural frequency, or alternatively at the maximum of the imaginary part of $\Delta \omega^{2}$. The generalized parameter $\eta_{r}=\eta_{r}\left(\omega_{r}\right)$ is in the following evaluated at the resonance frequency $\omega_{r}$ associated with the low-frequency stiffness of the viscoelastic material. When $\eta_{r}$ is separated into its real and imaginary part Eq. (15) can be written as

$$
\frac{\Delta \omega^{2}}{\Delta \omega_{r}^{2}}=\frac{\eta_{r}+\left|\eta_{r}\right|^{2}}{1+\left|\eta_{r}\right|^{2}+2 \operatorname{Re}\left[\eta_{r}\right]}
$$

with $\left|\eta_{r}\right|$ denoting the magnitude of the complex number. The imaginary part of Eq. (17) is then

$$
\operatorname{Im}\left[\frac{\Delta \omega^{2}}{\Delta \omega_{r}^{2}}\right]=\frac{\operatorname{Im}\left[\eta_{r}\right]}{1+\left|\eta_{r}\right|^{2}+2 \operatorname{Re}\left[\eta_{r}\right]}
$$

and it is seen to be proportional to the imaginary part of the generalized parameter. The maximum of the imaginary part in Eq. (18) is obtained by the variational relation $\delta\left(\operatorname{Im}\left[\Delta \omega^{2} / \Delta \omega_{r}^{2}\right]\right)=0$, which gives

$$
\delta\left(\operatorname{Im}\left[\eta_{r}\right]\right)\left(\left(1+\operatorname{Re}\left[\eta_{r}\right]\right)^{2}-\operatorname{Im}\left[\eta_{r}\right]^{2}\right)-\delta\left(\operatorname{Re}\left[\eta_{r}\right]\right)\left(1+\operatorname{Re}\left[\eta_{r}\right]\right) 2 \operatorname{Im}\left[\eta_{r}\right]=0
$$

The frequency function $G(\omega)$ represents the transition between the low- and high-frequency properties of the viscoelastic material defined in Eq. (1). For the standard three-parameter relaxation model in Fig. 1 the function $G(\omega) /(1-G(\omega))=i \omega \tau$ whereby the corresponding $\eta_{r}$ is purely imaginary. With respect to design considerations it is therefore reasonable to extend this property to general relaxation models, whereby optimality is only determined by the first term in Eq. (19) with $\delta \operatorname{Im}\left[\eta_{r}\right]$. Thus, the optimality relation (19) can be written as

$$
\operatorname{Im}\left[\eta_{r}\right]=1+\operatorname{Re}\left[\eta_{r}\right] \simeq 1
$$

where the latter approximation is again based on the assumption that the real part of the generalized parameter $\eta_{r}$ is negligible. The accuracy of this condition is demonstrated in the numerical example, considered next. 

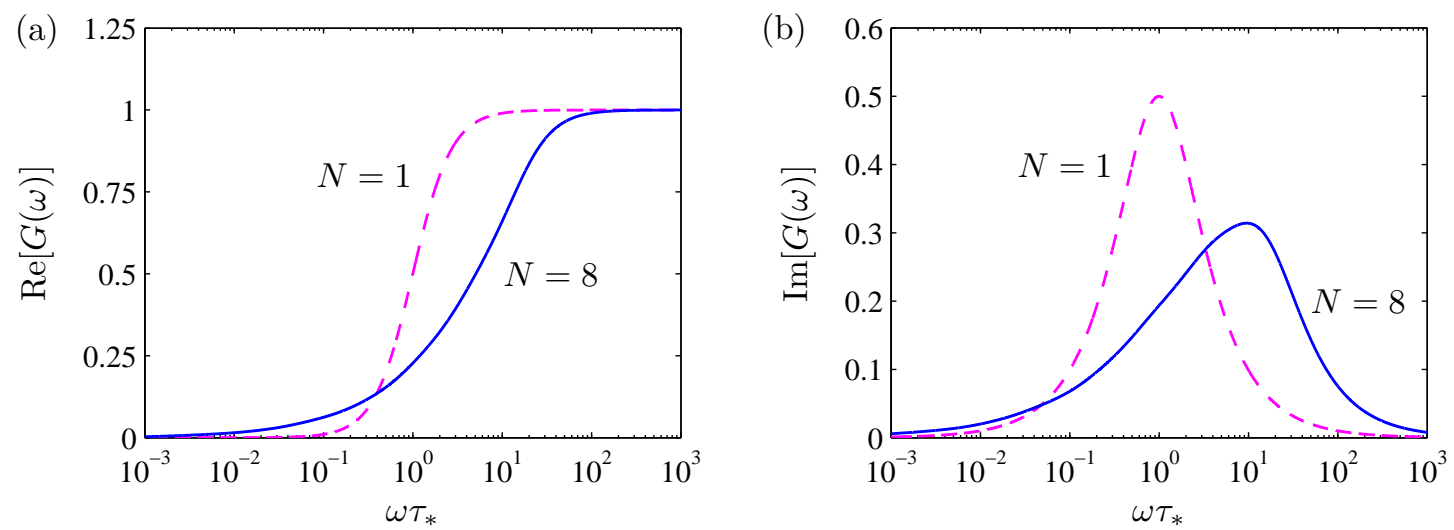

FiguRE 2. Real (a) and imaginary part (b) of frequency dependent function $G(\omega)$ for Prony series with $N=1$ (magenta - - ) and 8 (blue - - ).

\section{NumERICAL EXAMPLE}

In the present numerical example the accuracy of the explicit form of the frequency solution in Eq. (15) is illustrated in terms of a Prony series representation of the viscoelastic material properties. With respect to the model format in Eq. (1) the Prony series transfer function can be written as

$$
G(\omega)=\sum_{n=1}^{N} \alpha_{n} \frac{i \omega \tau_{n}}{1+i \omega \tau_{n}}
$$

where the high-frequency condition $G(\infty)=1$ implies $\sum \alpha_{n}=1$. Figure 2 shows the real and imaginary part of $G(\omega)$ for the relaxation parameters given by Slanik et al. [12], with assumed vanishing low-frequency stiffness $E_{0}=0$. For the eight term Prony series $(N=8)$ the parameters of $G(\omega)$ are reproduced in Table 1, while for the single-term representation ( $N=1)$ the time scale is $\tau_{1}=\tau_{*}$ and $\alpha_{1}=1$. Thus, the single term time scale $\tau_{*}$ is chosen as the reference time scale throughout this numerical example.

TABle 1. Eight term Prony series parameters from [12].

\begin{tabular}{lcccccccc}
\hline$n$ & 1 & 2 & 3 & 4 & 5 & 6 & 7 & 8 \\
$\tau_{j} / \tau_{*}$ & 0.0695 & 0.2771 & 1.1098 & 4.2017 & 18.856 & 70.097 & 348.64 & 1401.2 \\
$\alpha_{j}$ & 0.4574 & 0.2632 & 0.1553 & 0.0661 & 0.0356 & 0.0124 & 0.0070 & 0.0031 \\
\hline
\end{tabular}


(a)

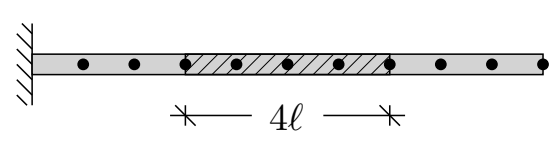

$* N_{v} \ell-*$

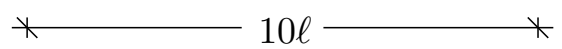

(b)

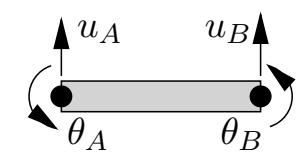

FiguRE 3. Cantilever beam with partial coverage by viscoelastic treatment.

In the case of a Prony series representation the combined equations of motion (2) and (4) are conveniently written in a linear state-space form with the associated eigenvalue problem $\mathbf{A} \mathbf{z}=i \omega \mathbf{z}$. The exact natural frequencies are therefore in the following determined as the eigenvalues $i \omega$ of the system matrix

$$
\mathbf{A}=\left[\begin{array}{ccccc}
\mathbf{0} & \mathbf{I} & \mathbf{0}_{H} & \ldots & \mathbf{0}_{H} \\
-\mathbf{M}^{-1} \mathbf{K} & -\mathbf{M}^{-1} \mathbf{C} & -\mathbf{M}^{-1} \mathbf{H} & \ldots & -\mathbf{M}^{-1} \mathbf{H} \\
\mathbf{0}_{H}^{T} & \alpha_{1} \Delta \mathbf{k} \mathbf{H}^{T} & -\mathbf{I}_{H H} / \tau_{1} & & \\
\vdots & \vdots & & \ddots & \\
\mathbf{0}_{H}^{T} & \alpha_{N} \Delta \mathbf{k} \mathbf{H}^{T} & & & -\mathbf{I}_{H H} / \tau_{N}
\end{array}\right]
$$

where $\mathbf{0}$ and $\mathbf{I}$ are the zero matrix and identity matrix in full dimensional space, $\mathbf{0}_{H}$ is a zero array of the same dimensions as the expansion array $\mathbf{H}$ and $\mathbf{I}_{H H}$ is the identity matrix in reduced dimensional space.

The flexible structure in the present example is the cantilever beam shown in Fig. 3(a) with partial treatment by the viscoelastic material with properties described in Table 1 . The beam structure is discretized by 10 simple beam elements of length $\ell$ and with transverse displacement and cross section rotation as the nodal degrees of freedom, see Fig. 3(b). The viscoelastic treatment covers four elements of the beam and it is placed after element $N_{v}$, as indicated by the hatched part in Fig. 3(a). In the present example the two locations corresponding to $N_{v}=2$ and 3 are considered.

In most beam vibration problems the viscoelastic treatment is introduced as internal shear layers in a sandwich configuration or in constrained layer damping [7]. However, in the 

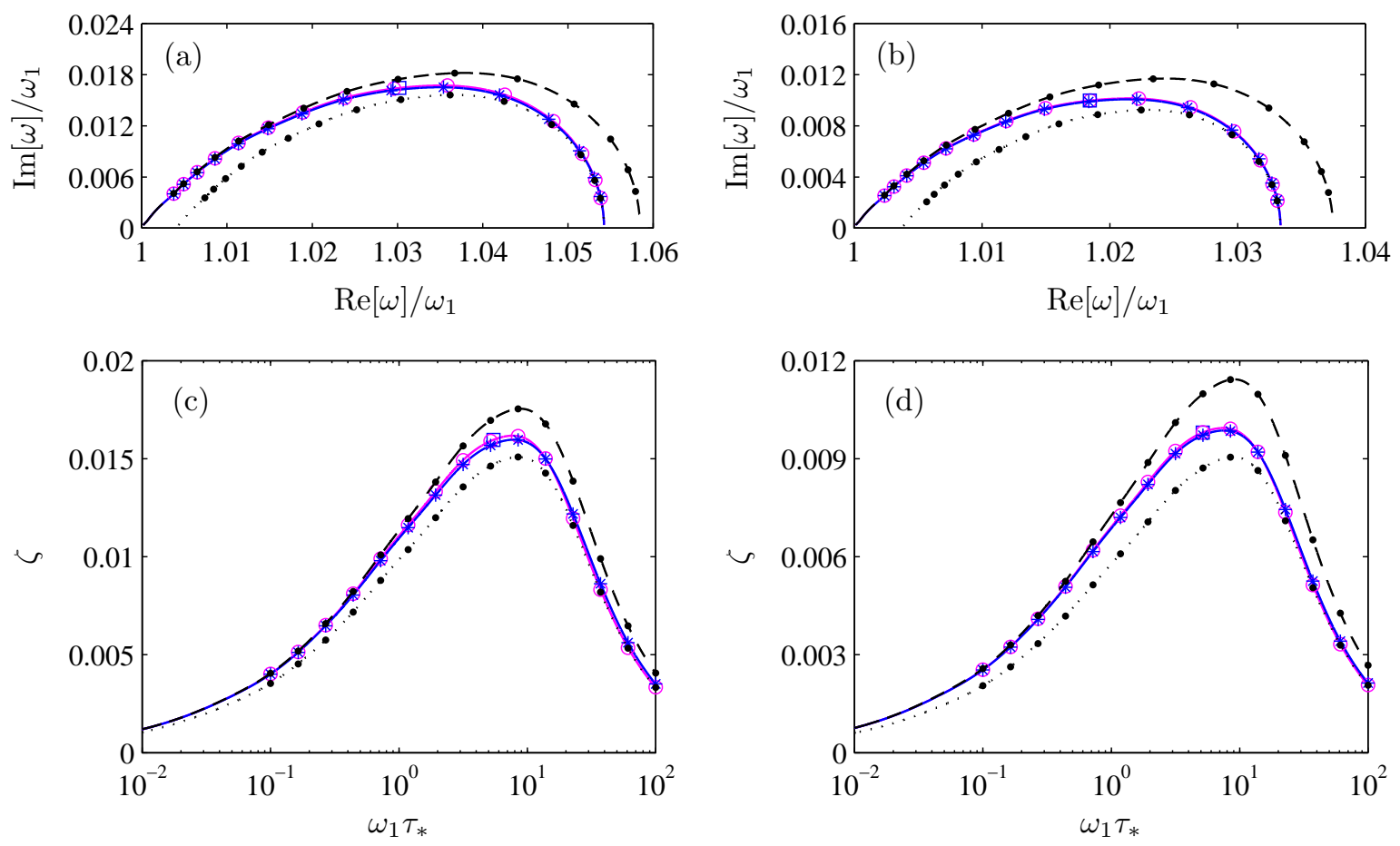

Figure 4. Natural frequency $(\mathrm{a}, \mathrm{b})$ and damping ratio $(\mathrm{c}, \mathrm{d})$ for first vibration mode $r=1$, and locations $N_{v}=2(\mathrm{a}, \mathrm{c})$ and $N_{v}=3(\mathrm{~b}, \mathrm{~d})$. Frequency solution (15) (blue $-*-$ ), exact solution (22) (magenta - o-), single-mode solutions (A.1) (black - - - - ) and (A.2) (black ……).

present numerical example the viscoelastic treatment is assumed to contribute to the bending stiffness of the beam, which therefore follows the relaxation form in Eq. (1)

$$
E I(\omega)=E I_{0}+\Delta E I G(\omega)
$$

where $G(\omega)$ is given by the Prony series representation in Eq. (21). The low-frequency bending stiffness $E I_{0}=0$, while the bending stiffness increment $\Delta E I=5 E I_{b}$, where $E I_{b}$ is the constant bending stiffness of the cantilever beam without the viscoelastic treatment. The contribution to the inertia from the viscoelastic treatment is omitted in this example. The generalized parameter $\eta_{r}$ in Eq. (16) contains a non-dimensional modal stiffness ratio, which is $\Delta k_{r} \omega_{r}^{2} /\left(k_{r} \Delta \omega_{r}^{2}\right)=1.128$ and 1.063 for $N_{v}=2$ and 3, respectively.

Figures 4(a) and (b) show the trajectories of the complex natural frequency for the first vibration mode of the structure $(r=1)$ for increasing $\tau_{*}$, while Figs. 4(c) and (d) present the corresponding damping ratio $\zeta=\operatorname{Im}[\omega] /|\omega|$. Figure 5 shows the same results for the 
second vibration mode of the structure $(r=2)$. The left figures (a) and (c) are for the treatment location $N_{v}=2$, whereas the figures (b) and (d) to the right present the results for the more indirect location $N_{v}=3$. The blue curves with asterisks $(*)$ represent the explicit solution in Eq. (15), while the magenta curves with circles (o) represent the exact natural frequency obtained as the eigenvalue of the matrix $\mathbf{A}$ in (22).

The black curves with bullets $(\bullet)$ represent the pure single mode solutions, described in Appendix A. The black dashed curves represent the solution in Eq. (A.1) obtained by modal expansion in terms of the mode shape vector $\mathbf{u}_{r}$ from the eigenvalue problem (5) with low-frequency stiffness contribution from the viscoelastic part. The black dotted curves represent the alternative solution in Eq. (A.2), where the modal expansion is based on the mode shape vector obtained from Eq. (6) with high-frequency stiffness contribution from the viscoelastic material. The two pure single mode solutions (black •) recover the individual real-valued solutions with $\tau_{*}=0$, while for increasing $\tau_{*}$ the error relative to the exact solution (magenta o) increases.

The explicit form of the frequency solution in Eq. (15) is seen to provide very accurate results over the full range of $\tau_{*}$. And by construction it recovers both real-valued limits and therefore reproduces the exact solutions with very good accuracy also in the intermediate range where the attainable damping is largest. The blue square markers ( $\square$ ) represent the exact natural frequency and damping ratio at the optimal value of $\tau_{*}$, obtained by the condition $\operatorname{Im}\left[\eta_{r}\right]=1$ derived in Eq. (20). In the present example for the first vibration form $\tau_{*}^{\mathrm{opt}} \omega_{1}=5.45$ and 5.15 for $N_{v}=2$ and 3 , respectively. For the second vibration mode the corresponding optimal time scales for $N_{v}=2$ and 3 are $\tau_{*}^{\text {opt }} \omega_{2}=5.13$ and 5.58, respectively. It is seen that $\tau_{*} \omega \simeq 5$ corresponds almost exactly to $\operatorname{Re}[G(\omega)]=\frac{1}{2}$ for $N=8$ in Fig. 2(a). For the damping ratio $\zeta$ the frequency solution in Eq. (15) also gives a very detailed reproduction of the exact damping ratio, and Eq. (15) may therefore be used for precise estimation of the damping level of flexible structures with partial viscoelastic treatment.

The computational cost associated with the frequency solution in Eq. (15) results from solving the two eigenvalue problems (5) and (6), while the cost associated with the exact solution comes directly from determining the eigenvalues of $\mathbf{A}$ in (22). In the present 
numerical example the eigenvalue solver eig in MATLAB has been used to determine all eigensolutions of the individual problems. It is found that the average computational cost in terms of CPU time is approximately 50 times larger when solving $\mathbf{A z}=i \omega \mathbf{z}$ compared to solving the two generalized eigenvalue problems in (5) and (6).
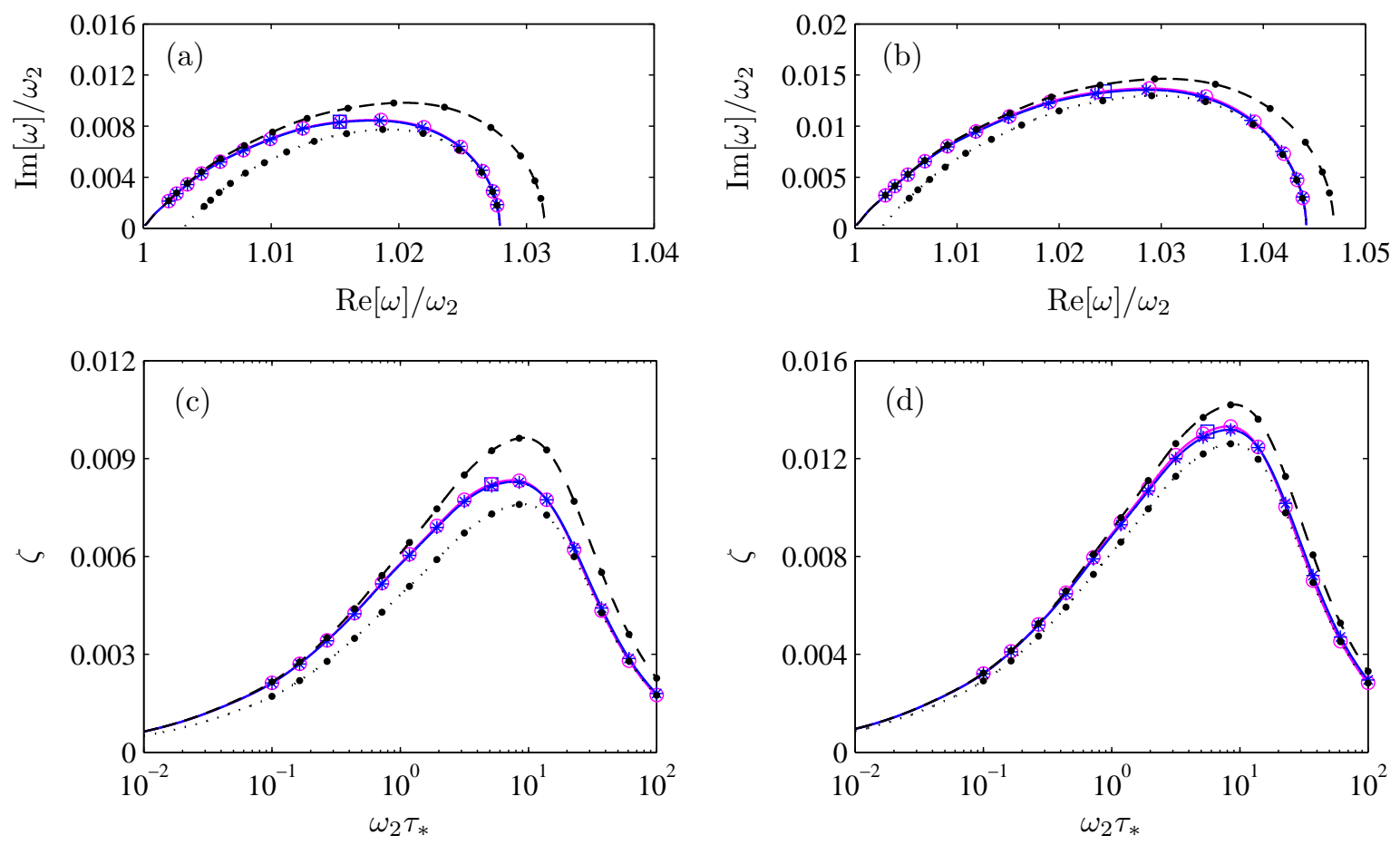

Figure 5. Natural frequency $(\mathrm{a}, \mathrm{b})$ and damping ratio $(\mathrm{c}, \mathrm{d})$ for second vibration mode $r=1$, and locations $N_{v}=2(\mathrm{a}, \mathrm{c})$ and $N_{v}=3(\mathrm{~b}, \mathrm{~d})$.

\section{Conclusions}

The free vibration properties of flexible damped structures are represented by the complexvalued natural frequencies, which are determined by solving the augmented eigenvalue problem in for example state-space format. The present paper presents a solution format for the natural frequency, which avoids the solution of the full eigenvalue problem, but instead requires the solution of the two real-valued (undamped) eigenvalue problems in Eqs. (5) and (6) associated with the low- and high-frequency stiffness limits of the viscoelastic treatment, respectively. The solutions to these real-valued eigenvalue problems are available in most commercial codes, while solving the complex eigenvalue problem is 
not necessarily feasible or possible. Furthermore, the present solution format is effective for design and optimization of viscoelastic damping of structures because the eigenvalue problem should not be solved when the frequency/time dependent properties are changed during an optimization routine. The solution format assumes that the constitutive relation of the viscoelastic material follows the relaxation format without energy dissipation in the low- and high-frequency limits, but seems otherwise to be applicable for general types of discretized structural models and viscoelastic treatment.

\section{Funding}

This work has been supported by Innovationsfonden Denmark via the project Power Pylons of the Future (PoPyFu).

\section{REFERENCES}

[1] S.-W. Kung and R. Singh, Vibration analysis of beams with multiple constrained layer damping patches, Journal of Sound and Vibration, vol. 212, pp. 781805, 1988.

[2] A.K. Lall, N.T. Asnani and B.C. Nakra, Damping analysis of partially covered sandwich beams, Journal of Sound and Vibration, vol. 123, pp. 247259, 1988.

[3] D.J. Mead and S. Markus, The forced vibration of a three-layer, damped sandwich beam with arbitrary boundary conditions, Journal of Sound and Vibration, vol. 10, pp. 163175, 1969.

[4] J.S. Moita, A.L. Araujo, P.G. Martins, C.M. Mota Soares and C.A. Mota Soares, Analysis of activepassive plate structures using a simple and efficient finite element model, Mechanics of Advanced Materials and Structures, vol. 18, pp. 159169, 2011.

[5] A. Benjeddou, Advances in hybrid active-passive vibration and noise control via piezoelectric and viscoelastic constrained layer treatments, Journal Vibration and Control, vol. 7, pp. 565602, 2001.

[6] N. Kumar and S.P. Singh, Vibration and damping characteristics of beams with active constrained layer treatments under parametric variations, Materials and Design, vol. 30, pp. 41624174, 2009.

[7] D. Granger and A. Ross, Effects of partial constrained viscoelastic layer damping parameters on the initial transient response of impacted cantilever beams: Experimental and numerical results, Journal of Sound and Vibration, vol. 321, pp. 45-64, 2009.

[8] C. Cai, H. Zheng and G.R. Liu, Vibration analysis of a beam with PCLD patch, Applied Acoustics, vol. 65 , pp. 1057-1076, 2004.

[9] S.C. Huang, D.J. Inman and E.M. Austin, Some design considerations for active and passive constrained layer damping treatments, Smart Materials and Structures, vol. 5, pp. 301313, 1996.

[10] Z.C. Xie and W.S. Shepard, Analytical modeling and analysis of a constrained layer damped plate to examine the impact of the transverse compressional damping component, Mechanics of Advanced Materials and Structures, vol. 21, pp. 669679, 2014.

[11] M. Bilasse, E.M. Daya and L. Azrar, Linear and nonlinear vibrations analysis of viscoelastic sandwich beams, Journal of Sound and Vibration, vol. 329, pp. 49504969, 2010.

[12] M.L. Slanik, J.A. Nemes, M.-J. Potvin and J.-C. Piedboeuf, Time domain finite element simulations of damped multilayered beams using a prony series representation, Mechanics of Time-Dependent Materials, vol. 4, pp. 211230, 2000. 
[13] Y. Wang and D.J. Inman, Finite element analysis and experimental study on dynamic properties of a composite beam with viscoelastic damping, Journal of Sound and Vibration, vol. 332, pp. 61776191, 2013.

[14] P.D. Cha, K.C. Carbon and R. Hsieh, Eigenvalues and eigenvalue sensitivities of a beam supported by viscoelastic solids, Journal of Vibration and Acoustics, vol. 136, 021017 (16pp), 2014.

[15] F. Boumediene, J.-M. Cadou, L. Duigou and E. Daya, A reduction model for eigensolutions of damped viscoelastic sandwich structures, Mechanics Research Communications, vol. 57, pp. 7481, 2014.

[16] M. Bilasse, L. Azrar and E.M. Daya, Complex modes based numerical analysis of viscoelastic sandwich plates vibrations, Computers and Structures, vol. 89, pp. 539555, 2010.

[17] R.L. Bisplinghoff and H. Ashley, Principles of Aeroelasticity, Wiley, New York, 1962.

[18] O.E. Hansteen and K. Bell, Accuracy of mode superposition analysis in structural dynamics, Earthquake Engineering and Structural Dynamics, vol. 7, pp. 405-411, 1979.

[19] R.L. Clark, Accounting for out-of-bndwidth modes in the assumed modes approach: Implications on colocated output feedback control, Journal of Dynamic Systems, Measurement, and Control, vol. 119, pp. 390-395, 1997.

[20] S. Krenk and J. Høgsberg, Tuned mass absorber on a flexible structure, Journal of Sound and Vibration, vol. 333, pp. 15771595, 2014.

[21] J. Høgsberg and S. Krenk, Balanced calibration of resonant piezoelectric RL shunts with quasi-static background flexibility correction, Submitted for publication, 2014.

[22] D.J. McTavish and P.C. Hughes, Modeling of linear viscoelastic space structures, Journal of Vibration and Acoustics, vol. 115, pp. 103110, 1993.

[23] D.M. Kochmann, Stable extreme damping in viscoelastic two-phase composites withnon-positivedefinite phases close to the loss of stability, Mechanics Research Communications, vol. 58, pp. 3645, 2014.

[24] S. Krenk, Damping mechanisms and models in structural dynamics. Proceedings of Structural Dynamics EURODYN 2002, Munich, Germany, pp. 87-98, Swets and Zeitlinger, Lisse, 2002.

[25] B. Lorenz, W. Pyckhout-Hintzen and B.N.J. Persson, Master curve of viscoelastic solid: Using causality to determine the optimal shifting procedure, and to test the accuracy of measured data, Polymer, vol. 55, pp. 565571, 2014.

[26] C. Zener, Elasticity and Anelasticity of Metals, The University of Chicago Press, Chicago, Illinois, 1948.

[27] J.A. Main and S. Krenk, Efficiency and tuning of viscous dampers on discrete systems, Journal of Sound and Vibration, vol. 286, pp. 97-122, 2005.

[28] J.R. Høgsberg and S. Krenk, Linear control strategies for damping of flexible structures, Journal of Sound and Vibration, vol. 293, pp. 59-77, 2006.

\section{Appendix A. Assumed single mode solutions}

The assumed single mode representation based on the eigenvalue problem (5) without correction for the flexibility from non-resonant modes is obtained by $1 / k_{0}=0$ in Eq. (12).

This gives

$$
\omega^{2}=\omega_{r}^{2}\left(1+G(\omega) \frac{\Delta k_{r}}{k_{r}}\right)
$$

which recovers $\omega=\omega_{r}$ for $G(0)=0$. 
Alternatively the frequency solution may be expressed in terms of the modal properties associated with the high-frequency stiffness limit of the viscoelastic treatment. Thus, in terms of the eigenvalue problem (6) the frequency solution can be written as

$$
\omega^{2}=\left(\omega_{r}^{2}+\Delta \omega_{r}^{2}\right)\left(1-(1-G(\omega)) \frac{\Delta k_{r}^{\infty}}{k_{r}^{\infty}}\right)
$$

where the natural frequency $\omega_{r}^{2}+\Delta \omega_{r}^{2}$ is associated with the high-frequency limit $G(\infty)=1$ and obtained as the eigenvalue of Eq. (6). The corresponding modal stiffness in Eq. (A.2) is defined as

$$
k_{r}^{\infty}=(\mathbf{u}+\Delta \mathbf{u})_{r}^{T}\left(\mathbf{K}+\mathbf{H} \Delta \mathbf{k H}^{T}\right)(\mathbf{u}+\Delta \mathbf{u})_{r}
$$

while the corresponding stiffness increment is

$$
\Delta k_{r}^{\infty}=(\mathbf{u}+\Delta \mathbf{u})_{r}^{T} \mathbf{H} \Delta \mathbf{k} \mathbf{H}^{T}(\mathbf{u}+\Delta \mathbf{u})_{r}
$$

The pure single mode solutions in Eqs. (A.1) and (A.2) are exact in the limits $\omega \tau \rightarrow 0$ and $\infty$, respectively, while in the intermediate frequency range they are approximations. 